\title{
Case of pleomorphic dermal sarcoma with systematic review of disease characteristics, outcomes and management
}

\author{
Andre Chu Qiao Lo 다, ${ }^{1}$ Sarah McDonald, ${ }^{2}$ Kai Yuen Wong $\mathbb{0}^{3}{ }^{3}$
}

${ }^{1}$ School of Clinical Medicine, University of Cambridge, Cambridge, UK ${ }^{2}$ Histopathology, Cambridge University Hospitals NHS Foundation Trust, Cambridge, UK

${ }^{3}$ Plastic \& Reconstructive Surgery, Cambridge University Hospitals NHS Foundation Trust, Cambridge, UK

Correspondence to Kai Yuen Wong: kaiyuen.wong@cantab.net

Accepted 22 August 2021

Check for updates

(C) BMJ Publishing Group Limited 2021. No commercial re-use. See rights and permissions. Published by BMJ.

To cite: Lo ACQ, McDonald S Wong KY. BMJ Case

Rep 2021;14:e244522.

doi:10.1136/bcr-2021-

244522

\section{DESCRIPTION}

A 79-year-old man presented with a 6-month history of four new painless enlarging scalp lesions. He had a strong history of sun exposure but otherwise no previous skin cancers. On examination, the lesions were suspicious for squamous cell carcinoma including a raised anterior scalp lesion measuring $4 \times 7 \mathrm{~mm}$ with an overlying crust (figure 1). The lesions were removed by standard margin excisions down to the periosteum and the areas reconstructed with split skin grafts.

Histological analyses revealed the anterior scalp lesion to be a pleomorphic dermal sarcoma (PDS). The other lesions were confirmed to be low risk squamous cell carcinoma and actinic keratosis. All lesions had clear margins ( $>1 \mathrm{~mm}$ ) except for the PDS, which had a close deep margin of $0.3 \mathrm{~mm}$ and demonstrated focal invasion to the galea, whereas peripheral margins were clear by around $4 \mathrm{~mm}$. Sections of the PDS showed a poorly circumscribed tumour, arising in sun-damaged skin, which expanded the dermis and infiltrated into and through the subcutis (figure 2). Tumour cells were variably epithelioid and spindled, with focal cytological atypia, including prominent nucleoli. Occasional multinucleated forms were observed and atypical mitoses were present. There was no evidence of tumour necrosis, nor lymphovascular or perineural invasion. Immunostaining was negative for MNF116, AE1/AE3, CK5/6, p63, desmin, S100, SOX10, CD34 and CD31. The immunohistochemical panel excluded other possible differential diagnoses, including squamous cell carcinoma, melanoma and angiosarcoma.

The patient was reviewed in a skin multidisciplinary team discussion and surgical re-excision was recommended for the PDS close deep margin, which he is currently awaiting.

PDS is characteristically a fast-growing, ulcerated, exophytic, bleeding skin tumour, similar to atypical fibroxanthomas, but with metastatic potential and is histologically distinguished by

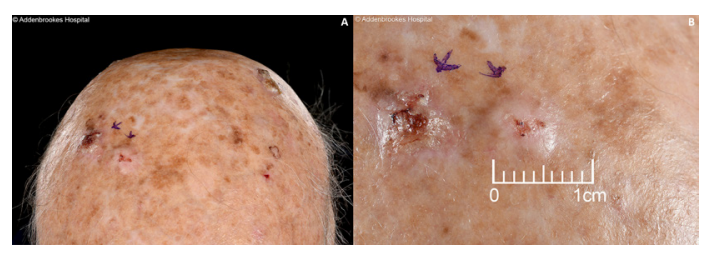

Figure 1 Pleomorphic dermal sarcoma on the anterior scalp ( $A$, right arrow) and close up ( $B$, right arrow).

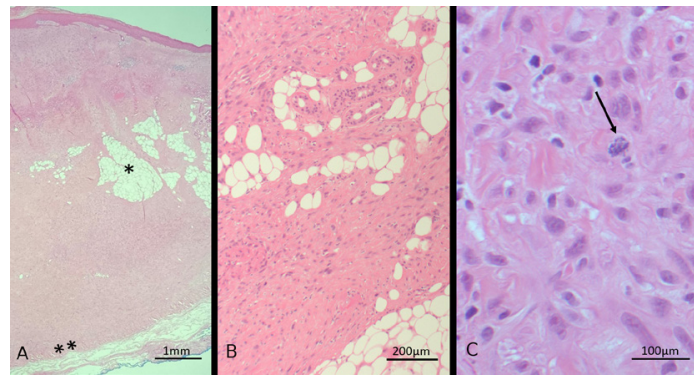

Figure 2 Histological features (H\&E) of the excised pleomorphic dermal sarcoma. (A) The tumour extends from the dermis, through the subcutaneous fat plane ${ }^{*}$, to focally invade galea aponeurotica** . (B) Tumour cells infiltrate among adipocytes in the subcutaneous fat. (C) Tumour cells exhibit pleomorphic, enlarged and irregular nuclei, with prominent nucleoli. Mitotic activity was identified (arrow), including occasional atypical mitotic figures.

necrosis, or deep subcutis, lymphovascular or perineural invasion. ${ }^{12}$ Little is known about the best clinical treatment due to its rarity. Hence, a systematic search in PubMed of "pleomorphic dermal sarcoma'” was conducted in April 2021, netting 134 articles, from which 15 were longitudinal studies that provided outcomes specific to PDS (table 1). ${ }^{2-16}$ Reported characteristics and outcomes (besides age) were pooled via randomeffects meta-analysis using the generalised linear mixed model with logit transformation, along with Clopper-Pearson CIs for individual studies. ${ }^{17}$ Heterogeneity was assessed via the $\mathrm{I}^{2}$ statistic and the likelihood-ratio test. ${ }^{18}$ Statistical tests were conducted using the meta package in $\mathrm{R}$ (V.3.6.0). ${ }^{19} 20$

In six studies, 238101214 PDS primarily affects older individuals (weighted mean age $=80.64$ ), and from meta-analysis, predominantly occurs in men $(82.80 \%, 95 \% \mathrm{CI}=72.37 \%$ to $89.95 \%$, $\mathrm{I}^{2}=62.1 \%$, likelihood-ratio test $\left.\mathrm{p}=0.011\right)$. Metaanalyses further showed that PDS is largely found on the scalp $(68.92 \%, 95 \% \mathrm{CI}=57.99 \%$ to $78.08 \%$, $\left.\mathrm{I}^{2}=54.9 \%, \mathrm{p}=0.019\right)$ in five studies, ${ }^{2} 381214$ or the face $(13.05 \%, 95 \% \mathrm{CI}=4.16 \%$ to $34.18 \%$, $\left.\mathrm{I}^{2}=88.1 \%, \mathrm{p}<0.0001\right)$ in six studies. ${ }^{2} 38101214$ Recurrence rate was $23.80 \%$ (95\% CI $=17.97 \%$ to $\left.30.82 \%, \mathrm{I}^{2}=11.5 \%, \mathrm{p}=0.037\right)$ in eight studies, ${ }^{2-4} 8-101214$ with $19.92 \%$ (95\% CI $=15.43 \%$ to $\left.25.32 \%, \mathrm{I}^{2}=0 \%, \mathrm{p}=0.050\right)$ local recurrence and $7.14 \%\left(95 \% \mathrm{CI}=4.55 \%\right.$ to $11.05 \%, \mathrm{I}^{2}=0 \%$, 
Images in...

Table 1 Studies included in systematic review

\begin{tabular}{|c|c|c|c|c|c|c|c|c|}
\hline $\begin{array}{l}\text { Study } \\
\text { (country) }\end{array}$ & $\mathrm{n}$ (male) & Mean size & Mean follow-up & Mean age & Location & $\begin{array}{l}\text { Local } \\
\text { recurrence }\end{array}$ & Metastases & Risk of bias* \\
\hline Bowe et $a l^{3}$ (UK) & $49(45)$ & $23.5 \mathrm{~mm}$ (median) & 22.4 months & 80 (median) & $\begin{array}{l}32 \text { scalp } \\
5 \text { forehead } \\
3 \text { ear } \\
3 \text { cheek } \\
2 \text { nose } \\
2 \text { lower leg } \\
1 \text { temple } \\
1 \text { lower lip }\end{array}$ & $\begin{array}{l}6 / 41 \\
\text { ( } 6 \text { clear margins) } \\
7 / 41\end{array}$ & $\begin{array}{l}\text { 1/41 } \\
\text { (1 death) }\end{array}$ & Moderate \\
\hline $\begin{array}{l}\text { Cesinaro et } a l^{4} \\
\text { (Italy) }\end{array}$ & 7 & - & 13 months & - & - & $\begin{array}{l}3 / 7 \\
3 / 7\end{array}$ & $\begin{array}{l}0 / 7 \\
\text { (0 deaths) }\end{array}$ & Low \\
\hline $\begin{array}{l}\text { Helbig et } a l^{5} \dagger \\
\text { (Germany) }\end{array}$ & $25(19)$ & - & - & 78 & $\begin{array}{l}3 \text { head/neck } \\
2 \text { shoulder }\end{array}$ & $\begin{array}{l}0 \text { deaths from } 1 \mathrm{~N} \\
\text { case with follow- }\end{array}$ & $\begin{array}{l}\text { AiTF-expressing PDS } \\
\text { up }\end{array}$ & High \\
\hline $\begin{array}{l}\text { Jasper et } a l^{6} \\
\text { (Canada) }\end{array}$ & 20 & - & - & - & - & $6 / 20$ & $2 / 20$ & Moderate \\
\hline $\begin{array}{l}\text { Klein et } \mathrm{al}^{7} \dagger \\
\text { (Germany) }\end{array}$ & $28(23)$ & - & - & 80 (median) & $\begin{array}{l}26 \text { head/neck } \\
2 \text { shoulder }\end{array}$ & $5 / 28$ & & Moderate \\
\hline $\begin{array}{l}\text { Lonie et al } \\
\text { (Australia) }\end{array}$ & $27(23)$ & - & 46.4 months & 79.27 & $\begin{array}{l}17 \text { scalp } \\
7 \text { face } \\
2 \text { ear } \\
1 \text { torso }\end{array}$ & $\begin{array}{l}2 / 26 \\
\text { (1 positive, } 1 \\
\text { close margin) } \\
3 / 27\end{array}$ & $\begin{array}{l}1 / 27 \\
\text { (1 death) }\end{array}$ & Low \\
\hline Miller et a/ ${ }^{8}$ (UK) & $32(27)$ & $25 \mathrm{~mm}$ & 24 months (median) & 81 & $\begin{array}{l}22 \text { scalp } \\
4 \text { forehead } \\
2 \text { ear } \\
2 \text { temple } \\
1 \text { eyebrow } \\
1 \text { forearm }\end{array}$ & $\begin{array}{l}8 / 29 \\
\text { ( } 7 \text { incomplete } \\
\text { removal) } \\
8 / 29\end{array}$ & $\begin{array}{l}3 / 29 \\
\text { ( } 0 \text { deaths) }\end{array}$ & Moderate \\
\hline Miller et al ${ }^{9}$ (USA) & 17 & - & - & - & - & $\begin{array}{l}2 / 2 \\
2 / 2\end{array}$ & $0 / 2$ & High \\
\hline $\begin{array}{l}\text { Müller et al }{ }^{10} \\
\text { (Germany) }\end{array}$ & $19(15)$ & $6.75 \mathrm{~cm}^{2}$ & - & 81.16 & $\begin{array}{l}16 \text { head } \\
2 \text { face } \\
1 \text { upper extremity }\end{array}$ & $\begin{array}{l}2 / 19 \\
2 / 19\end{array}$ & $0 / 19$ & High \\
\hline $\begin{array}{l}\text { Nonaka and } \\
\text { Bishop }{ }^{11} \text { (Japan) }\end{array}$ & 34 & - & $>2$ years & - & - & 1/34 death (positi & ve margin, metastasis) & Moderate \\
\hline $\begin{array}{l}\text { Persa et al }{ }^{12} \\
\text { (Europe) }\end{array}$ & $92(80)$ & $20 \mathrm{~mm}$ & 18 months & 81 (median) & $\begin{array}{l}76 \text { scalp } \\
9 \text { trunk } \\
7 \text { extremities }\end{array}$ & $\begin{array}{l}18 / 92 \\
26 / 92\end{array}$ & $8 / 92$ & Low \\
\hline $\begin{array}{l}\text { Ríos-Viñuela et } \\
a l^{13} \ddagger \text { (Spain) }\end{array}$ & $16(14)$ & $\begin{array}{l}25.63 \\
\mathrm{~mm}(n=15)\end{array}$ & 25.88 months & 80.43 & $\begin{array}{l}10 \text { scalp } \\
1 \text { forehead } \\
2 \text { nose } \\
2 \text { ear } \\
1 \text { temple }\end{array}$ & $\begin{array}{l}12 / 16 \text { ( } 8 \text { positive } \\
\text { margins) } \\
12 / 16\end{array}$ & 3/16 (2 deaths) & Low \\
\hline $\begin{array}{l}\text { Tardío et al }{ }^{14} \\
\text { (Spain) }\end{array}$ & $18(9)$ & $22 \mathrm{~mm}$ & 40 months & 81 & $\begin{array}{l}9 \text { scalp } \\
5 \text { forehead } \\
1 \text { nose } \\
1 \text { eyebrow } \\
1 \text { cheek } \\
1 \text { temple }\end{array}$ & $\begin{array}{l}3 / 15 \\
(3 \text { positive } \\
\text { margins) } \\
5 / 15\end{array}$ & $\begin{array}{l}3 / 15 \\
\text { (3 deaths) }\end{array}$ & Moderate \\
\hline $\begin{array}{l}\text { Thum et } a l^{15} \S \\
\text { (UK) }\end{array}$ & $3(3)$ & $\begin{array}{l}31.67 \\
\mathrm{~mm}\end{array}$ & 3 months & 79 & $\begin{array}{l}2 \text { scalp } \\
1 \text { temple }\end{array}$ & \multicolumn{2}{|c|}{$\begin{array}{l}0 \text { recurrences or deaths from } 2 \\
\text { pseudovascular PDS cases with follow- } \\
\text { up }\end{array}$} & Moderate \\
\hline $\begin{array}{l}\text { Wang et } a l^{16} \text { ๆ } \\
\text { (USA) }\end{array}$ & $6(6)$ & $11 \mathrm{~mm}$ & 48.6 months & 61 & $\begin{array}{l}3 \text { scalp } \\
2 \text { temple } \\
1 \text { ear }\end{array}$ & \multicolumn{2}{|c|}{$\begin{array}{l}4 \text { recurrences and } 4 \text { deaths from } 6 \\
\text { metastatic PDS cases }\end{array}$} & Low \\
\hline Total & 331 (199/237 male) & $\begin{array}{l}21.47 \\
\mathrm{~mm}\end{array}$ & 13-46.4 months & 80.64 & $\begin{array}{l}156 / 218 \text { scalp } \\
33 / 237 \text { face }\end{array}$ & $\begin{array}{l}50 / 251 \\
56 / 232\end{array}$ & $\begin{array}{l}18 / 252 \\
\text { (6/174 deaths) }\end{array}$ & $\begin{array}{l}5 \text { low } \\
7 \text { moderate } \\
3 \text { high }\end{array}$ \\
\hline
\end{tabular}

* Rated using the modified Newcastle-Ottawa Scale for case series from Haffar et al. ${ }^{21}$

tResults not pooled with others due to overlap with cohort from Persa et al. ${ }^{12}$

‡Results not pooled with others as study cohort was primarily composed of recurrent PDS referred from other institutions.

$\S$ Results not pooled with others as study examines a subset cohort from Miller et $a l^{8}$ of pseudovascular PDS.

IResults not pooled with others as study examined metastatic PDS only.

MiTF, Microphthalmia-associated transcription factor; PDS, pleomorphic dermal sarcoma.

0.261) metastasis over nine studies (mean follow-up 13-46.4 months). ${ }^{2-4} 68-101214$ Among four studies, ${ }^{2} 381487.25 \%$ $\left(95 \% \mathrm{CI}=3.11 \%\right.$ to $\left.99.93 \%, \mathrm{I}^{2}=91.0 \%, \mathrm{p}=0.0003\right)$ of local recurrences were due to incomplete removal or inadequate margins. There is limited evidence showing radiotherapy is effective. $^{2} 612$ There is also limited evidence showing modified Mohs micrographic surgery offers better prognosis than conventional surgery. ${ }^{13}$ Conversely, margins $<2 \mathrm{~cm}$ predispose worse prognosis. ${ }^{12}$ Two metastatic cases were successfully managed by chemotherapy. ${ }^{28}$ 


\section{Learning points}

- Pleomorphic dermal sarcoma is a rare cancer that presents primarily in older men on sun-exposed areas such as the scalp.

- Due to the metastatic potential of pleomorphic dermal sarcoma, it is important not to miss this diagnosis and to ensure complete resection of the tumour.

\section{Twitter Andre Chu Qiao Lo @iamandrelo}

Contributors ACQL, SM and KYW drafted the case report. ACQL conducted the systematic review and meta-analysis. ACQL, SM and KYW critically revised and approved the final manuscript.

Funding The authors have not declared a specific grant for this research from any funding agency in the public, commercial or not-for-profit sectors.

Competing interests None declared.

Patient consent for publication Obtained.

Provenance and peer review Not commissioned; externally peer reviewed.

\section{ORCID iDs}

Andre Chu Qiao Lo http://orcid.org/0000-0001-7339-6809

Kai Yuen Wong http://orcid.org/0000-0002-6060-1487

\section{REFERENCES}

1 Iglesias-Pena N, Martínez-Campayo N, López-Solache L. Relation between atypical Fibroxanthoma and pleomorphic dermal sarcoma: histopathologic features and review of the literature. Actas Dermosifiliogr 2021;112:392-405.

2 Lonie S, Yau B, Henderson M, et al. Management of pleomorphic dermal sarcoma. ANZ J Surg 2020;90:2322-4.

3 Bowe CM, Godhania B, Whittaker M, et al. Pleomorphic dermal sarcoma: a clinical and histological review of 49 cases. Br J Oral Maxillofac Surg 2021;59:460-5.

4 Cesinaro AM, Gallo G, Tramontozzi S, et al. Atypical fibroxanthoma and pleomorphic dermal sarcoma: a reappraisal. J Cutan Pathol 2021;48:207-10.

5 Helbig D, Mauch C, Buettner R, et al. Immunohistochemical expression of melanocytic and myofibroblastic markers and their molecular correlation in atypical fibroxanthomas and pleomorphic dermal sarcomas. J Cutan Pathol 2018:45:880-5.
6 Jasper KD, Holloway CL, DeVries KJ, et al. Local relapse and survival outcomes in patients with scalp sarcoma: a retrospective study of 95 patients treated in a provincial cancer care institution over 25 years. Cureus 2019;11.

7 Klein S, Quaas A, Noh K-W, et al. Integrative analysis of pleomorphic dermal sarcomas reveals fibroblastic differentiation and susceptibility to immunotherapy. Clin Cancer Res 2020;26:5638-45.

8 Miller K, Goodlad JR, Brenn T. Pleomorphic dermal sarcoma: adverse histologic features predict aggressive behavior and allow distinction from atypical fibroxanthoma. Am J Surg Pathol 2012;36:1317-26.

9 Miller TI, Zoumberos NA, Johnson B, et al. A genomic survey of sarcomas on sun-exposed skin reveals distinctive candidate drivers and potentially targetable mutations. Hum Pathol 2020;102:60-9.

10 Müller CSL, Kreie L, Bochen F, et al. Expression of 3q oncogene SEC62 in atypical fibroxanthoma-immunohistochemical analysis of 41 cases and correlation with clinical, viral and histopathologic features. Oncol Lett 2019;17:1768-76.

11 Nonaka D, Bishop PW. Sarcoma-like tumor of head and neck skin. Am J Surg Pathol 2014;38:956-65

12 Persa OD, Loquai C, Wobser M, et al. Extended surgical safety margins and ulceration are associated with an improved prognosis in pleomorphic dermal sarcomas. J Eur Acad Dermatol Venereol 2019;33:1577-80.

13 Ríos-Viñuela E, Serra-Guillén C, Llombart B, et al. Pleomorphic dermal sarcoma: a retrospective study of 16 cases in a dermato-oncology centre and a review of the literature. Eur J Dermatol 2020;30:545-53.

14 Tardío JC, Pinedo F, Aramburu JA, et al. Pleomorphic dermal sarcoma: a more aggressive neoplasm than previously estimated. J Cutan Pathol 2016;43:101-12.

15 Thum C, Husain EA, Mulholland K, et al. Atypical fibroxanthoma with pseudoangiomatous features: a histological and immunohistochemical mimic of cutaneous angiosarcoma. Ann Diagn Pathol 2013;17:502-7.

16 Wang W-L, Torres-Cabala C, Curry JL, et al. Metastatic atypical fibroxanthoma: a series of 11 cases including with minimal and NO subcutaneous involvement. Am J Dermatopathol 2015;37:455-61.

17 Clopper CJ, Pearson ES. The use of confidence or fiducial limits illustrated in the case of the binomial. Biometrika 1934;26:404-13.

18 Hartley HO, Rao JN. Maximum-Likelihood estimation for the mixed analysis of variance model. Biometrika 1967;54:93-108.

19 Balduzzi S, Rücker G, Schwarzer G. How to perform a meta-analysis with R: a practical tutorial. Evid Based Ment Health 2019;22:153-60.

20 R Core Team. R: a language and environment for statistical computing. Vienna, Austria: R Foundation for Statistical Computing, 2019. https://www.R-project.org/

21 Haffar S, Bazerbachi F, Prokop L, et al. Frequency and prognosis of acute pancreatitis associated with fulminant or non-fulminant acute hepatitis A: a systematic review. Pancreatology 2017;17:166-75.

Copyright 2021 BMJ Publishing Group. All rights reserved. For permission to reuse any of this content visit https://www.bmj.com/company/products-services/rights-and-licensing/permissions/

BMJ Case Report Fellows may re-use this article for personal use and teaching without any further permission.

Become a Fellow of BMJ Case Reports today and you can:

- Submit as many cases as you like

- Enjoy fast sympathetic peer review and rapid publication of accepted articles

- Access all the published articles

Re-use any of the published material for personal use and teaching without further permission

Customer Service

If you have any further queries about your subscription, please contact our customer services team on +44 (0) 2071111105 or via email at support@bmj.com.

Visit casereports.bmj.com for more articles like this and to become a Fellow 